\title{
Incompressibility of neutron-rich matter
}

\author{
J. Piekarewicz ${ }^{1, *}$ and M. Centelles ${ }^{2,}$ \\ ${ }^{1}$ Department of Physics, Florida State University, Tallahassee, Florida 32306, USA \\ ${ }^{2}$ Departament d'Estructura i Constituents de la Matèria and Institut de Ciències del Cosmos, Facultat de Física, \\ Universitat de Barcelona, Diagonal 647, E-08028 Barcelona, Spain
}

(Received 24 December 2008; published 12 May 2009)

\begin{abstract}
The saturation properties of neutron-rich matter are investigated in a relativistic mean-field formalism using two accurately calibrated models: NL3 and FSUGold. The saturation properties-density, binding energy per nucleon, and incompressibility coefficient—are calculated as a function of the neutron-proton asymmetry $\alpha \equiv(N-Z) / A$ to all orders in $\alpha$. Good agreement (at the $10 \%$ level or better) is found between these numerical calculations and analytic expansions that are given in terms of a handful of bulk parameters determined at saturation density. Using insights developed from the analytic approach and a general expression for the incompressibility coefficient of infinite neutron-rich matter, i.e., $K_{0}(\alpha)=K_{0}+K_{\tau} \alpha^{2}+\cdots$, we construct a hybrid model with values for $K_{0}$ and $K_{\tau}$ as suggested by recent experimental findings. Whereas the hybrid model provides a better description of the measured distribution of isoscalar monopole strength in the Sn isotopes relative to both NL3 and FSUGold, it significantly underestimates the distribution of strength in ${ }^{208} \mathrm{~Pb}$. Thus, we conclude that the incompressibility coefficient of neutron-rich matter remains an important open problem.
\end{abstract}

DOI: 10.1103/PhysRevC.79.054311

PACS number(s): 21.65.Mn, 21.65.Ef, 21.60.Jz, 21.30.Fe

\section{INTRODUCTION}

The incompressibility of neutron-rich matter remains at the forefront of both experimental and theoretical investigations because of its fundamental role in constraining the equation of state (EOS) of cold dense matter. The incompressibility coefficient characterizes the small-density fluctuations of infinite nuclear matter around its equilibrium point, thereby providing the first glimpse into the "stiffness" of the equation of state.

It is widely accepted that the giant monopole resonance (GMR) - the quintessential compressional mode-provides the cleanest, most direct route to the nuclear incompressibility. In a procedure first proposed by Blaizot and collaborators [1,2], finite nuclei GMR energies and the nuclear-matter incompressibility should both be computed within the same theoretical framework. This procedure avoids altogether the reliance on macroscopic ("liquid-drop-like") approaches that have proven unreliable for the extraction of the incompressibility coefficient of infinite nuclear matter from finite nuclei GMR energies [3,4]. In most theoretical approaches, an accurately calibrated model is obtained by fitting the model parameters to a set of selected ground-state properties of finite nuclei. In some recent instances, excited states-computed as the consistent linear response of the mean-field ground state-have also been incorporated into the fit [5]. If such accurately calibrated models are able to reproduce the experimental distribution of monopole strength (or at least some of its moments), then the value of the incompressibility coefficient predicted by the model is regarded as reliable. Following this procedure, it has been established that the incompressibility coefficient of symmetric nuclear matter falls within the following relatively narrow range: $K_{0}=240 \pm 10 \mathrm{MeV}[6,7]$.

\footnotetext{
*jpiekarewicz@fsu.edu
}

†mariocentelles@ub.edu
Because of the collective nature of the GMR, a strong coherent peak develops only in the case of relatively heavy nuclei. Indeed, the monopole strength in "light" nuclei, such as ${ }^{40} \mathrm{Ca}$, is strongly fragmented. As (stable) heavy nuclei are characterized by a significant neutron excess, experimental studies of the GMR probe the incompressibility of neutron-rich matter rather than that of symmetric matter. As such, GMR energies on a variety of nuclei having different neutron-proton asymmetries $[\alpha \equiv(N-Z) / A]$, such as ${ }^{90} \mathrm{Zr},{ }^{116} \mathrm{Sn},{ }^{144} \mathrm{Sm}$, and ${ }^{208} \mathrm{~Pb}$, provide simultaneous constraints on the incompressibility of symmetric nuclear matter $\left(K_{0}\right)$ as well as on its leading $\alpha$ correction, a quantity that will be denoted by $K_{\tau}$. That is, the incompressibility of infinite neutron-rich matter may be parametrized to leading order in the neutron-proton asymmetry as $K_{0}(\alpha)=K_{0}+K_{\tau} \alpha^{2}$. It is therefore natural to assume that previous lessons learned in the case of $K_{0}$ will remain relevant for $K_{\tau}$. First and foremost, $K_{\tau}$ should not be inferred from an extrapolation to the $A \rightarrow \infty$ limit from laboratory experiments on finite nuclei. Rather, one should continue to follow the procedure advocated by Blaizot and demand that the values of both $K_{0}$ and $K_{\tau}$ be those predicted by a consistent theoretical model that successfully reproduces the experimental GMR energies of a variety of nuclei. We note that in the present contribution, both $K_{0}$ and $K_{\tau}$ refer to bulk properties of the infinite system.

It is therefore the aim of the present manuscript to (a) use accurately calibrated relativistic mean-field models to extract the saturation properties of infinite neutron-rich matter, (b) compute GMR energies for a variety of nuclei using the consistent isoscalar-monopole response of the mean-field ground state, and (c) confront these theoretical results against experimental GMR energies-especially the new data on tin $[6,7]$. As a byproduct of this procedure, analytic approaches to the saturation properties of infinite neutron-rich matter based on a few bulk parameters calculated at saturation density will be validated against exact numerical results. 
The manuscript has been organized as follows. In Sec. II a brief review of the relativistic mean-field formalism will be provided. Particular emphasis will be placed on developing a thorough description of the properties of infinite neutron-rich matter and on the various bulk parameters that define its behavior around nuclear-matter saturation density. In Sec. III results will be presented for the evolution of the saturation point with neutron-proton asymmetry using both exact (numerical) and approximate (analytic) approaches. We finish this section by revisiting the topic of why tin is so soft [6-10]. Our summary and conclusions will follow in Sec. IV.

\section{FORMALISM}

The starting point for the calculation of various ground-state properties is an interacting Lagrangian density of the following form:

$$
\begin{aligned}
\mathscr{L}_{\text {int }}= & \bar{\psi}\left[g_{s} \phi-\left(g_{v} V_{\mu}+\frac{g_{\rho}}{2} \boldsymbol{\tau} \cdot \mathbf{b}_{\mu}+\frac{e}{2}\left(1+\tau_{3}\right) A_{\mu}\right) \gamma^{\mu}\right] \psi \\
& -\frac{\kappa}{3 !}\left(g_{s} \phi\right)^{3}-\frac{\lambda}{4 !}\left(g_{s} \phi\right)^{4}+\frac{\zeta}{4 !}\left(g_{v}^{2} V_{\mu} V^{\mu}\right)^{2} \\
& +\Lambda_{v}\left(g_{\rho}^{2} \mathbf{b}_{\mu} \cdot \mathbf{b}^{\mu}\right)\left(g_{v}^{2} V_{\mu} V^{\mu}\right) .
\end{aligned}
$$

The Lagrangian density includes an isodoublet nucleon field $(\psi)$ interacting via the exchange of two isoscalar mesons, a scalar $(\phi)$ and a vector $\left(V^{\mu}\right)$, one isovector meson $\left(b^{\mu}\right)$, and the photon $\left(A^{\mu}\right)[11,12]$. In addition to meson-nucleon interactions, the Lagrangian density is supplemented by nonlinear meson interactions with coupling constants denoted by $\kappa, \lambda, \zeta$, and $\Lambda_{v}$, which are responsible for a softening of the nuclear-matter equation of state, both for symmetric-nuclear and pure-neutron matter. Whereas $\kappa$ and $\lambda$ are instrumental in the softening of the equation of state of symmetric nuclear matter near saturation density, $\zeta$ softens the equation of state but at higher densities. Finally, the mixed vector coupling $\Lambda_{v}$ [13] has been introduced to soften the density dependence of the symmetry energy - a quantity that is predicted to be stiff in most relativistic mean-field models. This effective Lagrangian has been used to compute a variety of ground-state observables at the mean-field level and will be used here to study the incompressibility of neutron-rich matter. Further details on the calibration and implementation of the relativistic meanfield models may be found in Refs. [11-14] and references therein.

Asymmetric nuclear matter is an idealized system consisting of an infinite number of neutrons and protons interacting exclusively through the nuclear force. At zero temperature and in the thermodynamic limit (where both the baryon number $A=N+Z$ and the volume of the system $V$ tend to infinity), the binding energy per nucleon depends solely on two intensive variables: the baryon density $\rho \equiv A / V$ and the neutron-proton asymmetry $\alpha \equiv(N-Z) / A$ (the latter may be expressed as $\alpha=\left(\rho_{n}-\rho_{p}\right) / \rho$ in terms of the nucleon densities). By studying such an idealized system, one hopes to elucidate how the volume and symmetry terms of the semiempirical mass formula [15] evolve with density.
It has become customary to write the energy per particle of infinite nuclear matter as

$$
\begin{aligned}
E / A(\rho, \alpha)-M \equiv & \mathcal{E}(\rho, \alpha)=\mathcal{E}_{\mathrm{SNM}}(\rho) \\
& +\alpha^{2} \mathcal{S}_{2}(\rho)+\alpha^{4} \mathcal{S}_{4}(\rho)+\cdots,
\end{aligned}
$$

where we have indicated that $E / A$ is measured relative to the nucleon rest mass $M$. As the neutron-proton asymmetry is constrained to the interval $0 \leqslant \alpha \leqslant 1$, the total energy per particle $\mathcal{E}(\rho, \alpha)$ is often expanded in a power series in $\alpha^{2}$. Note that odd powers of $\alpha$ do not contribute to the expansion owing to the symmetry of the strong force between like-nucleon pairs. The leading term in this expansion, i.e., $\mathcal{E}_{\mathrm{SNM}}(\rho) \equiv \mathcal{E}(\rho, \alpha=$ $0)$, represents the contribution from symmetric $(N=Z=$ $A / 2)$ matter. The leading $\mathcal{O}\left(\alpha^{2}\right)$ correction to the symmetric limit, i.e., $\mathcal{S}_{2}(\rho) \equiv \mathcal{S}(\rho)$, is known as the symmetry energy. The contribution $\alpha^{2} \mathcal{S}(\rho)$ thus measures the energy involved in converting part of the protons in symmetric nuclear matter to excess neutrons, at total baryon density $\rho$. The above power-series expansion is particularly useful as the symmetry energy dominates the corrections to the symmetric limit for all values of $\alpha$. Indeed, to an excellent approximation, the energy per particle of pure neutron matter $(\alpha \equiv 1)$ may be written as

$$
\mathcal{E}_{\mathrm{PNM}}(\rho) \equiv \mathcal{E}(\rho, \alpha=1) \approx \mathcal{E}_{\mathrm{SNM}}(\rho)+\mathcal{S}(\rho) .
$$

The main feature that we aim to understand in the present manuscript is the evolution with neutron-proton asymmetry of the bulk properties of infinite nuclear matter-such as the saturation density, the binding energy at saturation, and the incompressibility coefficient. Particularly important is to characterize the sensitivity of the results to the density dependence of the symmetry energy. To do so and to establish a baseline, we start by describing the behavior of symmetric nuclear matter near saturation density.

\section{A. Symmetric nuclear matter}

One of the hallmarks of nuclear dynamics is the saturation of symmetric $(\alpha \equiv 0)$ infinite nuclear matter. The saturation point is characterized by an equilibrium density of about $\rho_{0} \simeq 0.15 \mathrm{fm}^{-3}$ and an energy per particle of $\varepsilon_{0} \simeq-16 \mathrm{MeV}$. Given that the pressure $P=\rho^{2} \partial \mathcal{E}_{\mathrm{SNM}} / \partial \rho$ of symmetric nuclear matter vanishes at saturation, then the small density fluctuations around the saturation point are fully characterized by the incompressibility coefficient $K_{0}$. Yet for reasons that will become clear later, the behavior of symmetric nuclear matter is expanded in a Taylor series up to third order in the small density fluctuations. That is,

$$
\mathcal{E}_{\mathrm{SNM}}(\rho)=\varepsilon_{0}+\frac{1}{2} K_{0} x^{2}+\frac{1}{6} Q_{0} x^{3}+\cdots,
$$

where $x$ is a conveniently defined dimensionless parameter that characterizes the deviations of the density from its saturation value, i.e.,

$$
x \equiv \frac{\left(\rho-\rho_{0}\right)}{3 \rho_{0}} .
$$

The various bulk coefficients that characterize the behavior of the symmetric system near saturation density are given as 
follows:

$$
\begin{aligned}
\varepsilon_{0} & =\mathcal{E}_{\mathrm{SNM}}(x=0)=\mathcal{E}_{\mathrm{SNM}}\left(\rho=\rho_{0}\right), \\
K_{0} & =\mathcal{E}_{\mathrm{SNM}}^{\prime \prime}(x=0)=9 \rho_{0}^{2}\left(\frac{\partial^{2} \mathcal{E}_{\mathrm{SNM}}}{\partial \rho^{2}}\right)_{\rho=\rho_{0}}, \\
Q_{0} & =\mathcal{E}_{\mathrm{SNM}}^{\prime \prime \prime}(x=0)=27 \rho_{0}^{3}\left(\frac{\partial^{3} \mathcal{E}_{\mathrm{SNM}}}{\partial \rho^{3}}\right)_{\rho=\rho_{0}} .
\end{aligned}
$$

\section{B. Symmetry energy}

In the so-called parabolic approximation, the deviations from the symmetric $(\alpha \equiv 0)$ limit are controlled by the $\mathcal{O}\left(\alpha^{2}\right)$-symmetry energy [see Eq. (2)]. As has been done for the symmetric case, the behavior of the symmetry energy around nuclear-matter saturation density may be conveniently characterized in terms of a few bulk parameters, namely,

$$
\mathcal{S}(\rho)=J+L x+\frac{1}{2} K_{\mathrm{sym}} x^{2}+\frac{1}{6} Q_{\mathrm{sym}} x^{3}+\cdots,
$$

where $J, L, K_{\mathrm{sym}}$, and $Q_{\text {sym }}$ are the values of the symmetry energy, slope, curvature, and third derivative at saturation density. However, unlike symmetric nuclear matter, the "symmetry pressure" $L$ does not vanish. Indeed, the symmetry pressure-a quantity that strongly influences the neutron-skin thickness of heavy nuclei-is (within the parabolic approximation) directly proportional to the pressure of pure neutron matter. That is,

$$
P_{0}=\frac{1}{3} \rho_{0} L .
$$

A one-parameter fit to the low-density behavior of the symmetry energy that is frequently used in the heavy-ion community is of the following form [16-22]:

$$
\mathcal{S}(\rho)=\mathcal{S}_{0}\left(\frac{\rho}{\rho_{0}}\right)^{\gamma}=J(1+3 x)^{\gamma},
$$

where in arriving at the last term we have made use of Eqs. (5) and (7). To the extent that the above parametrization is accurate, the following relations should be satisfied:

$$
\begin{aligned}
L & =\left(\frac{\partial \mathcal{S}}{\partial x}\right)_{x=0}=3 J \gamma, \\
K_{\text {sym }} & =\left(\frac{\partial^{2} \mathcal{S}}{\partial x^{2}}\right)_{x=0}=9 J \gamma(\gamma-1), \\
P_{0} & =\rho_{0} J \gamma .
\end{aligned}
$$

With due caution, mainly because the connection of heavy-ion experiments to the EOS is not at all trivial and often involves model-dependent extrapolations of the measured data, significant constraints on the value of the coefficient $\gamma$ have been extracted in the last few years from different experimental observables. For instance, in intermediate-energy heavy-ion collisions, the analysis of isoscaling data [20,21] provides a $\gamma$ value around 0.69 . Transport model simulations of data related to isospin diffusion favor the milder constraint $\gamma \sim 0.69-1.05$ $[19,22]$. Some nuclear collective modes provide another tool for probing the behavior of $\mathcal{S}(\rho)$ at subsaturation densities. The values $P_{0}=2.3 \pm 0.8 \mathrm{MeV} / \mathrm{fm}^{3}$ and $J=32 \pm 1.8 \mathrm{MeV}$ extracted in Ref. [23] from pygmy dipole resonances suggest a value of $\gamma \sim 0.5 \pm 0.15$, whereas the constraint $23.3<$ $\mathcal{S}\left(\rho=0.1 \mathrm{fm}^{-3}\right)<24.9 \mathrm{MeV}$ obtained in Ref. [24] from the properties of the giant dipole resonance in ${ }^{208} \mathrm{~Pb}$ hints at a value of $\gamma \sim 0.5-0.65$. These findings from experimental isospin-sensitive signals imply a rather soft nuclear symmetry energy at subsaturation densities. An analysis [25] of a set of neutron skins of nuclei measured across the mass table by antiprotonic techniques yields a similar conclusion. Finally, recent studies of the low-density behavior of pure neutron matter using universal properties of dilute Fermi gases seem to support the same findings [26,27].

\section{Neutron-rich matter}

Insofar as neutron-rich matter saturates, the energy per particle may continue to be written as in Eq. (4). Thus, the aim of this section is to characterize the evolution of the saturation point-in particular, the saturation density, the binding energy per nucleon, and the incompressibility coefficient-as a function of the neutron-proton asymmetry $\alpha=(N-Z) / A$. To do so, it will prove instructive to proceed along two alternative paths: one purely analytical and the other purely numerical. In the analytic case, the saturation properties of neutron-rich matter will be derived from a handful of bulk parameters that characterize the behavior of both symmetric nuclear matter and the symmetry energy around saturation density, as was done in Eqs. (4) and (7). This purely analytic procedure, already well known in the literature, will be contrasted against a numerical procedure that is free of any assumptions or approximations beyond that of the mean-field approximation. We will verify that these two alternative approaches agree at the few percent level, thereby lending support to the analytic approach in elucidating the evolution of the incompressibility coefficient with neutron excess.

According to Eqs. (4) and (7), the energy per particle of asymmetric nuclear matter with a neutron-proton asymmetry $\alpha$ may be written in the form

$$
\begin{aligned}
\mathcal{E}(\rho, \alpha) \approx & \left(\varepsilon_{0}+J \alpha^{2}\right)+L \alpha^{2} x+\frac{1}{2}\left(K_{0}+\alpha^{2} K_{\text {sym }}\right) x^{2} \\
& +\frac{1}{6}\left(Q_{0}+\alpha^{2} Q_{\text {sym }}\right) x^{3} .
\end{aligned}
$$

Clearly, the presence of the linear term shifts the saturation point from $x_{0} \equiv 0$ to $\bar{x}_{0}$, where the latter is defined as the physical solution to the following equation:

$$
\begin{aligned}
\left(\frac{\partial \mathcal{E}}{\partial x}\right)= & \alpha^{2} L+\left(K_{0}+\alpha^{2} K_{\text {sym }}\right) x \\
& +\frac{1}{2}\left(Q_{0}+\alpha^{2} Q_{\text {sym }}\right) x^{2}=0 .
\end{aligned}
$$

Although the roots of this equation may be found by solving a simple quadratic equation, the $\mathcal{O}\left(\alpha^{2}\right)$ solution may be solved by inspection. One obtains

$$
\begin{aligned}
\bar{x}_{0} & =-\frac{L}{K_{0}} \alpha^{2}+\mathcal{O}\left(\alpha^{4}\right) \quad \text { or } \\
\bar{\rho}_{0} / \rho_{0} & =1+3 \bar{x}_{0}=1-3 \frac{L}{K_{0}} \alpha^{2}+\mathcal{O}\left(\alpha^{4}\right) .
\end{aligned}
$$

The values for the energy per particle and the incompressibility coefficient may now be found by expanding Eq. (11) around 
TABLE I. Model parameters used in the calculations. The parameter $\kappa$ and the meson masses $m_{s}, m_{v}$, and $m_{\rho}$ are all given in $\mathrm{MeV}$. The value of the nucleon mass is taken as $M=939 \mathrm{MeV}$.

\begin{tabular}{lcccccccccc}
\hline \hline Model & $m_{s}$ & $m_{v}$ & $m_{\rho}$ & $g_{s}^{2}$ & $g_{v}^{2}$ & $g_{\rho}^{2}$ & $\kappa$ & $\lambda$ & $\zeta$ & $\Lambda_{v}$ \\
\hline FSU & 491.500 & 782.500 & 763.000 & 112.1996 & 204.5469 & 138.4701 & 1.4203 & +0.02376 & 0.06 & 0.03 \\
NL3 & 508.194 & 782.501 & 763.000 & 104.3871 & 165.5854 & 79.6000 & 3.8599 & -0.01591 & 0.00 & 0.00 \\
Hybrid & 508.194 & 782.501 & 763.000 & 106.2575 & 165.5848 & 79.6483 & 4.5472 & -0.01952 & 0.00 & 0.00 \\
\hline \hline
\end{tabular}

this new $(\alpha \neq 0)$ value of the saturation density:

$$
\mathcal{E}(\rho, \alpha)=\mathcal{E}\left(\bar{x}_{0}, \alpha\right)+\frac{1}{2}\left(x-\bar{x}_{0}\right)^{2} \mathcal{E}^{\prime \prime}\left(\bar{x}_{0}, \alpha\right)+\cdots .
$$

Alternatively, by introducing the dimensionless parameter

$$
\bar{x} \equiv \frac{\left(\rho-\bar{\rho}_{0}\right)}{3 \bar{\rho}_{0}}
$$

to characterize the deviations of the density from the new saturation point, the expansion of $\mathcal{E}(\rho, \alpha)$ given in Eq. (14) may be cast in a form analogous to Eq. (4) for $\mathcal{E}_{\mathrm{SNM}}(\rho)$. That is,

$$
\begin{aligned}
\mathcal{E}(\rho, \alpha) & =\mathcal{E}\left(\bar{x}_{0}, \alpha\right)+\frac{1}{2}\left[\left(1+3 \bar{x}_{0}\right)^{2} \mathcal{E}^{\prime \prime}\left(\bar{x}_{0}, \alpha\right)\right] \bar{x}^{2}+\cdots \\
& \equiv \varepsilon_{0}(\alpha)+\frac{1}{2} K_{0}(\alpha) \bar{x}^{2}+\cdots
\end{aligned}
$$

where the energy per particle and the incompressibility coefficient at the new saturation density are given by

$$
\begin{aligned}
\varepsilon_{0}(\alpha) & \equiv \mathcal{E}\left(\bar{x}_{0}, \alpha\right)=\varepsilon_{0}+J \alpha^{2}+\mathcal{O}\left(\alpha^{4}\right), \\
K_{0}(\alpha) & \equiv\left(1+3 \bar{x}_{0}\right)^{2} \mathcal{E}^{\prime \prime}\left(\bar{x}_{0}, \alpha\right) \\
& =K_{0}+\left(K_{\text {sym }}-6 L-\frac{Q_{0}}{K_{0}} L\right) \alpha^{2}+\mathcal{O}\left(\alpha^{4}\right) .
\end{aligned}
$$

The analytic results correct to second order in $\alpha$ are summarized in the following set of equations, where the quantities $\rho_{\tau}, \varepsilon_{\tau}$, and $K_{\tau}$ represent the deviations of the saturation density, energy per particle, and incompressibility coefficient of infinite matter away from the symmetric $N=$ $Z$ limit:

$$
\begin{aligned}
\rho_{0}(\alpha) & =\rho_{0}+\rho_{\tau} \alpha^{2}+\mathcal{O}\left(\alpha^{4}\right)=\rho_{0}-3 \rho_{0} \frac{L}{K_{0}} \alpha^{2}+\mathcal{O}\left(\alpha^{4}\right), \\
\varepsilon_{0}(\alpha) & =\varepsilon_{0}+\varepsilon_{\tau} \alpha^{2}+\mathcal{O}\left(\alpha^{4}\right)=\varepsilon_{0}+J \alpha^{2}+\mathcal{O}\left(\alpha^{4}\right) \\
K_{0}(\alpha) & =K_{0}+K_{\tau} \alpha^{2}+\mathcal{O}\left(\alpha^{4}\right) \\
& =K_{0}+\left(K_{\text {sym }}-6 L-\frac{Q_{0}}{K_{0}} L\right) \alpha^{2}+\mathcal{O}\left(\alpha^{4}\right)
\end{aligned}
$$

In view of the profuse choices of terminology existing in the literature, our notation and conventions are discussed further in the Appendix.

\section{RESULTS}

Having developed the necessary formalism in the preceding sections, we devote this section to presenting the results of our calculations. As we have done elsewhere [8], we generated our results using two accurately calibrated models: NL3 [28,29] and FSUGold [5]. In addition, we performed calculations with a hybrid model to be introduced later. Effective meson masses (i.e., interaction ranges) and coupling constants for the present models are displayed in Table I as defined by the Lagrangian density of Eq. (1).

With the above sets of parameters, one may compute the nuclear-matter equation of state, namely, the energy per particle as a function of density and neutron excess. In particular, one can extract values for the various bulk parameters defined in Eqs. (4) and (7) that characterize the behavior of neutron-rich matter around saturation density; these parameters are listed in Table II. Note that to make contact with the parametrization given in Eq. (9), the value of the exponent $\gamma$ listed in Table II was extracted from a fit to the symmetry energy in the low-density range of $\rho=(0.3-1.0) \rho_{0}$. The found results are in consonance with the prediction $\gamma=L / 3 J$ that follows from Eq. (9) (namely, $\gamma=0.62$ for FSUGold and $\gamma=1.06$ in the case of NL3 and the hybrid model). Whereas the FSUGold and NL3 models agree on the energy and density at saturation-quantities that are tightly constrained by existing ground-state observablessignificant discrepancies emerge in all remaining parameters. The main difference between the two models may be succinctly summarized by stating that whereas FSUGold predicts a soft behavior for both symmetric nuclear matter (through $K_{0}$ ) and the symmetry energy (through $L$ ), NL3 predicts a stiff behavior for both. Note that "stiff" and "soft" refer to whether the energy increases rapidly or slowly, respectively, with density.

That symmetric nuclear matter and the symmetry energy are either both soft (as in the FSUGold model) or both stiff (as in the NL3 model) may lie at the core of the problem in reproducing the experimentally measured GMR energies in the Sn isotopes [6-8]. According to Eq. (18c), a large value of $L$ (as in NL3) generates a large softening of the incompressibility

TABLE II. Bulk parameters (as described in the text) characterizing the energy of symmetric nuclear matter [Eq. (4)] and the symmetry energy [Eq. (7)] at saturation density. All quantities are in MeV, with the exception of $\rho_{0}$ given in $\mathrm{fm}^{-3}$ and the dimensionless parameter $\gamma$ defined in Eq. (9).

\begin{tabular}{lccccccrc}
\hline \hline Model & $\rho_{0}$ & $\varepsilon_{0}$ & $K_{0}$ & $Q_{0}$ & $J$ & \multicolumn{1}{c}{$L$} & \multicolumn{1}{c}{$K_{\text {sym }}$} & $\gamma$ \\
\hline FSU & 0.148 & -16.30 & 230.0 & -523.4 & 32.59 & 60.5 & -51.3 & 0.64 \\
NL3 & 0.148 & -16.24 & 271.5 & +204.2 & 37.29 & 118.2 & +100.9 & 0.98 \\
Hybrid & 0.148 & -16.24 & 230.0 & -71.5 & 37.30 & 118.6 & +110.9 & 0.98 \\
\hline \hline
\end{tabular}


coefficient relative to its value in symmetric nuclear matter. However, a large incompressibility coefficient in symmetric nuclear matter $K_{0}$ (as in NL3) hinders the softening generated by $K_{\tau}$. Conversely, FSUGold predicts a relatively small value for $K_{0}$. However, its soft symmetry energy generates a small (absolute) value for $K_{\tau}$ that precludes the significant reduction in the incompressibility coefficient required by the experimental GMR energies. In an effort to circumvent this problem — and this problem only_we have generated a hybrid model having the same incompressibility coefficient as FSUGold while preserving the stiff symmetry energy of NL3 (see Table II). As seen in Table I, this was accomplished through a slight adjustment of the scalar self-coupling parameters $\kappa$ and $\lambda$. Note that it is not our intent to accurately calibrate the hybrid model introduced here. Rather, the hybrid modelalthough reasonably accurate-should merely be regarded as a "test" model that illustrates how surprisingly soft are the experimental GMR energies of the tin isotopes relative to the theoretical predictions. Indeed, as we will display later in Fig. 8, not even such an artificially tuned model can fully account for the rapid softening of the GMR energies with neutron excess. Let us mention that nowadays it is generally acknowledged that experimental data on compressional-mode giant resonances support a value of $K_{0} \approx 240 \mathrm{MeV}$ [30] for the incompressibility coefficient of symmetric nuclear matter. Recently measured data on the breathing mode of $\mathrm{Sn}$ isotopes seem to favor a constraint $K_{\tau}=-550 \pm 100 \mathrm{MeV}$ for the asymmetry term in the nuclear incompressibility [6,7]. A similar value of $K_{\tau} \sim-500 \mathrm{MeV}$ has been reported from independent experimental evidence available from isospin diffusion observables in nuclear reactions [19,22] and from neutron skins of nuclei [25]. Tables II and III confirm that the hybrid model is consistent with both of the indicated $K_{0}$ and $K_{\tau}$ values

The evolution of the equation of state with neutron-proton asymmetry $\alpha$ is displayed in Fig. 1 for the three models considered in the text: FSUGold (solid blue lines), NL3 (dashed green lines), and hybrid (dot-dashed red lines). The $\alpha=0$ curve corresponds to symmetric nuclear matter, whereas the $\alpha=1$ curve corresponds to pure neutron matter. In all models, nuclear matter ceases to saturate at a value of the neutron-proton asymmetry slightly larger than $\alpha=0.75$. Figure 2 provides an expanded version of the results for symmetric nuclear matter $(\alpha=0)$ and for pure neutron matter $(\alpha=1)$. To an excellent approximation-especially at the subsaturation densities of relevance to this work-the

TABLE III. Leading $\mathcal{O}\left(\alpha^{2}\right)$ correction to the evolution of the saturation density, energy per particle, and incompressibility coefficient of asymmetric nuclear matter. Quantities outside the parentheses were extracted from a quadratic fit to the numerical results in the $0 \leqslant \alpha^{2} \leqslant 0.1$ range, whereas the quantities in parentheses were computed from the analytic expressions given in Eqs. (18).

\begin{tabular}{lccc}
\hline \hline Model & $\rho_{\tau}\left(\mathrm{fm}^{-3}\right)$ & $\varepsilon_{\tau}(\mathrm{MeV})$ & $K_{\tau}(\mathrm{MeV})$ \\
\hline FSU & $-0.117(-0.117)$ & $32.60(32.59)$ & $-275.45(-276.77)$ \\
NL3 & $-0.188(-0.194)$ & $37.24(37.29)$ & $-682.65(-697.36)$ \\
Hybrid & $-0.215(-0.229)$ & $37.20(37.30)$ & $-531.98(-563.86)$ \\
\hline \hline
\end{tabular}

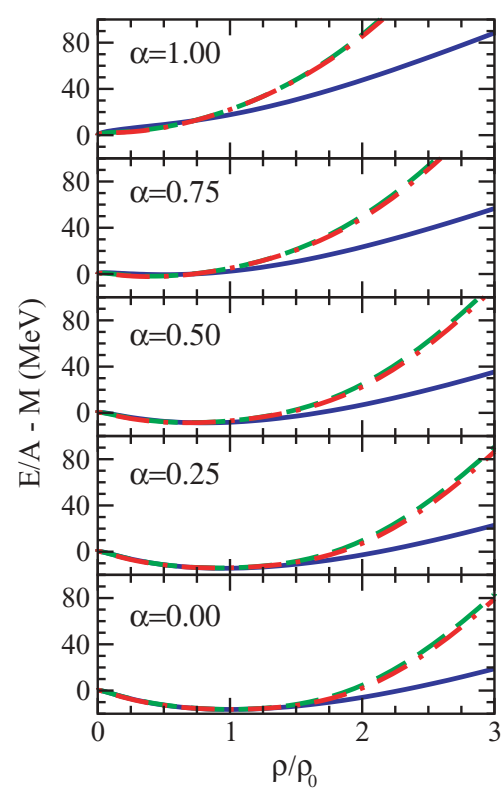

FIG. 1. (Color online) Density dependence of the energy per particle in infinite nuclear matter at the shown neutron-proton asymmetries according to the FSUGold (blue solid line), NL3 (green dashed line), and hybrid (red dot-dashed line) models.

difference between the equation of state of pure neutron matter and that of symmetric matter equals the symmetry energy of Fig. 3. On the other hand, a truncated expansion $\mathcal{S}(\rho)=J+L x+\frac{1}{2} K_{\text {sym }} x^{2}$ [cf. Eq. (7)] appears to provide a fair enough representation of the actual value of $\mathcal{S}(\rho)$ in a range of densities roughly between half and twice the saturation density of symmetric nuclear matter. Indeed, we find that the discrepancies of this approximation compared with the exact $\mathcal{S}(\rho)$ are less than $5 \%$ in a density range $0.45 \rho_{0} \lesssim \rho \lesssim 2.7 \rho_{0}$ for FSUGold and $0.33 \rho_{0} \lesssim \rho \lesssim 2.15 \rho_{0}$ for NL3 and the hybrid model.

One observes that in all cases, the hybrid model seems to follow closely the predictions of the NL3 model in Figs. 1-3. For the symmetry energy, this has been done by construction.

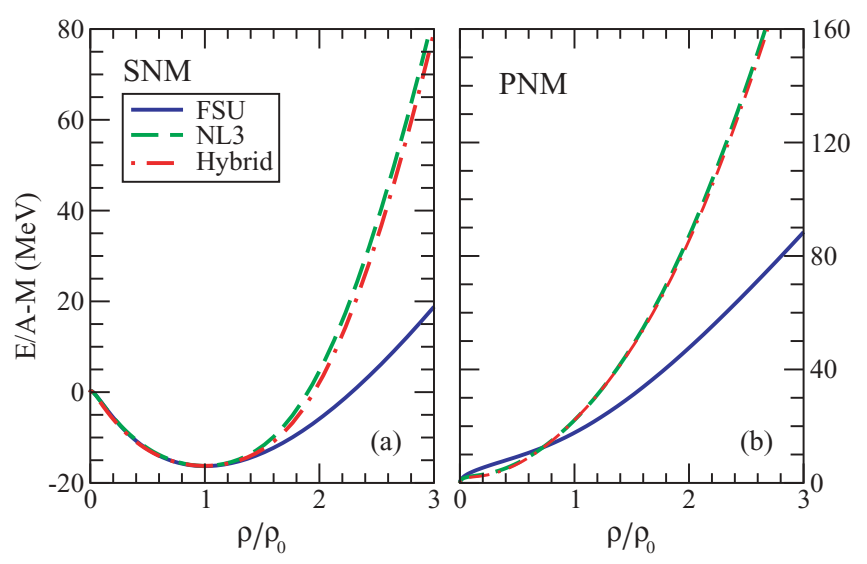

FIG. 2. (Color online) Density dependence of the energy per particle in symmetric nuclear matter (SNM) and in pure neutron matter (PNM) for the investigated models. 


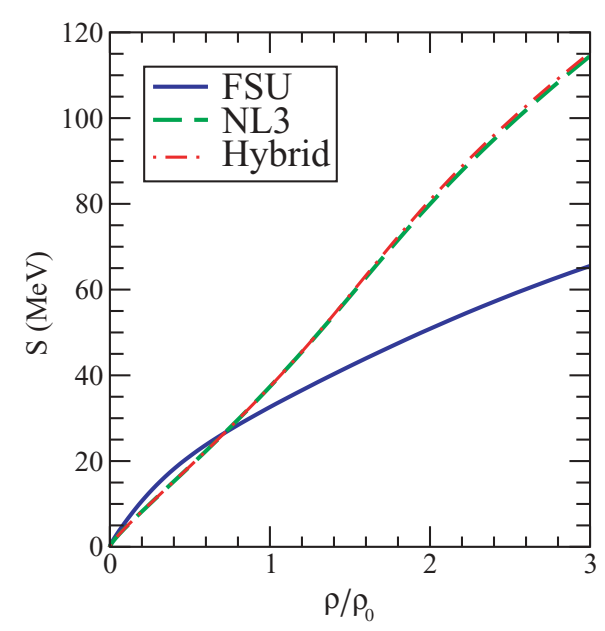

FIG. 3. (Color online) Density dependence of the symmetry energy coefficient for the investigated models.

For symmetric nuclear matter, however, the hybrid model is indeed softer than NL3-and as soft as FSUGold-at saturation density. That the hybrid model tracks NL3 at high density is a reflection of the vector self-coupling parameter $\zeta$ having been set to zero in both models. This confirms that the value of the incompressibility coefficient of symmetric nuclear matter at saturation density has practically no impact on the equation of state of high-density matter and, by extension, on most neutron-star properties [31].

From the evolution of the equation of state with $\alpha$, we conclude quite generally that as the neutron-proton asymmetry increases, the saturation density is reduced, the binding energy weakens, and the nuclear incompressibility softens. Based on the structure of Eqs. (18), we regard these trends as model independent. Indeed, that the saturation density is reduced (i.e., $\rho_{\tau}<0$ ) is a simple reflection of (a) symmetric nuclear matter has a stable equilibrium point $\left(K_{0}>0\right)$ and (b) the pressure of pure neutron matter at saturation density is positive $(L>0)$. Further, that the binding energy weakens (i.e., $\varepsilon_{\tau}>0$ ) follows from the fact that pure neutron matter is not self-bound, namely, $J \geqslant\left|\epsilon_{0}\right|$. Finally, that the nuclear incompressibility softens requires $K_{\tau}<0$. This is the hardest condition to satisfy as it depends on higher-order derivatives of the equation of state, namely, on $Q_{0}$ and $K_{\text {sym }}$ [see Eq. (18c)]. However, barring anomalously large values for these two quantities, the condition $K_{\tau}<0$ hinges also on the pressure of pure neutron matter at saturation density being positive. This is due to the large coefficient multiplying $L$ in Eq. (18c), which provides the dominant contribution to $K_{\tau}$ as compared to $K_{\text {sym }}$ and $Q_{0} / K_{0}$. We conclude that whereas the signs of $\rho_{\tau}, \varepsilon_{\tau}$, and $K_{\tau}$ are fairly model independent, their model-dependent magnitudes are determined by two fundamental parameters of the equation of state: the incompressibility coefficient of symmetric nuclear matter $K_{0}>0$ and the symmetry pressure $L$.

To examine the evolution of the saturation point with neutron-proton asymmetry, we have tabulated in Table III the values for $\rho_{\tau}, \varepsilon_{\tau}$, and $K_{\tau}$. These quantities (which have been enclosed in parentheses) were computed directly from the analytic expressions given in Eqs. (18). The bulk parameters that they depend on were previously extracted from a fit to the equation of state of symmetric nuclear matter and to the symmetry energy and are listed in Table II. As alluded earlier, the fact that neither $Q_{0}$ nor $K_{\text {sym }}$ are anomalously large in the present models results in an asymmetry term in the nuclear incompressibility $K_{\tau}$ that is dominated by the symmetry pressure $L$. In particular, note that the value of $K_{\tau}$ in the hybrid model is consistent with the value extracted in Refs. [6,7] from the measurement of the GMR energies in the $\mathrm{Sn}$ isotopes.

But how accurate are the expressions given in Eqs. (18)? To test the reliability of these analytic expressions, we have carried out a purely numerical exercise that is exact within the purview of the mean-field approximation. There is no reliance on the parabolic approximation, as in Eq. (2), or on expansions around the saturation density of symmetric matter, such as in Eq. (11). Basically, the equation of state of asymmetric nuclear matter is computed numerically for a range of values of the neutron-proton asymmetry in the $0 \leqslant \alpha^{2} \leqslant 0.1$ range. For each value of $\alpha$, the new saturation point-namely the density, energy per nucleon, and incompressibility coefficient at the minimum -is computed. Once this procedure is completed, one extracts the three desired coefficients $\left(\rho_{\tau}, \varepsilon_{\tau}\right.$, and $\left.K_{\tau}\right)$ from a least-squares fit to the numerical data. Such a procedure is illustrated in Figs. 4-6 for the saturation density, energy per particle, and incompressibility coefficient, respectively. In all cases, the inset includes a comparison between the exact numerical results (displayed with lines) and the analytic approximations (displayed with symbols). Moreover, $\rho_{\tau}, \varepsilon_{\tau}$, and $K_{\tau}$ have also been tabulated in Table III. The agreement between the analytic and numerical results is fairly good (at worst the discrepancies amount to $\sim 6 \%$ ) suggesting that arguments based on Eqs. (18), which imply an expansion in both $\alpha$ and the density around the saturation point of $N=Z$ matter, are not only insightful but also quantitatively accurate.

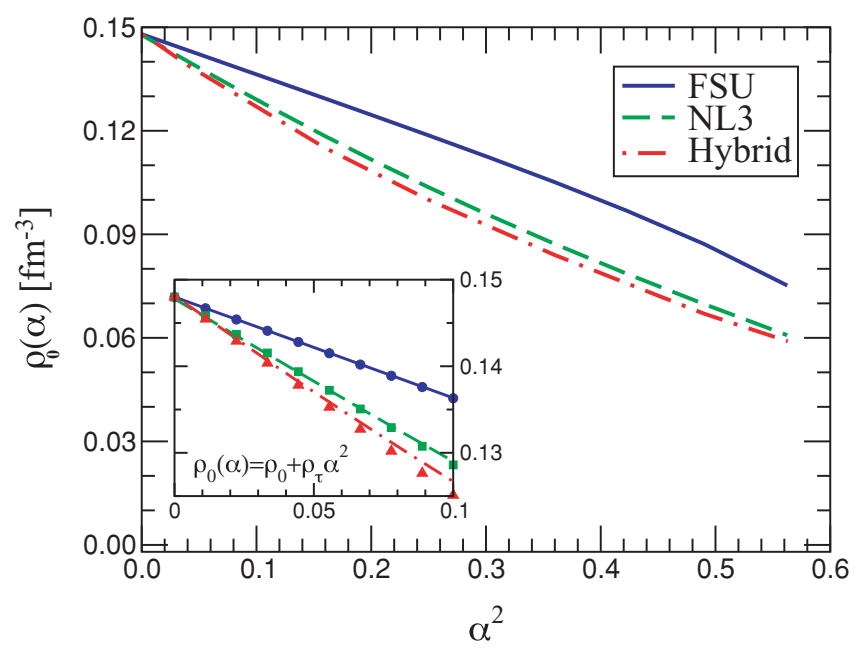

FIG. 4. (Color online) Evolution with increasing neutron-proton asymmetry of the baryon density that corresponds to the exact saturation point of asymmetric nuclear matter. The inset displays by symbols the result of a least-squares fit in the $0 \leqslant \alpha^{2} \leqslant 0.1$ range assuming a parabolic dependence. 


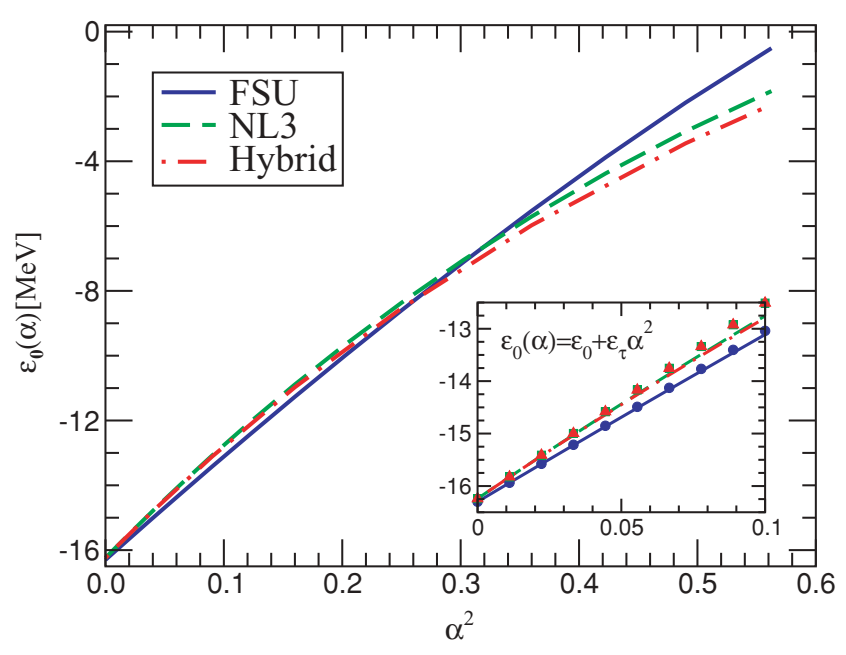

FIG. 5. (Color online) Same as Fig. 4, but for the energy per particle.

The goodness of the parabolic approximation for the binding energy of asymmetric matter seems to be confirmed also in other frameworks such as microscopic many-body calculations $[32,33]$ or model analyses of the symmetry energy coefficient in nucleus-nucleus collisions [34].

We finish this section by revisiting a topic recently addressed in the literature: Why is tin so soft? [6-10]. Namely, GMR energies of even- $A$ isotopes of tin from $A=112$ to $A=$ 124 measured in a recent experiment [6,7] are significantly lower than the values predicted with accurately calibrated, otherwise successful, mean-field models. Note that the same models satisfactorily predict the GMR excitation energy of ${ }^{90} \mathrm{Zr}$ and ${ }^{208} \mathrm{~Pb}$. Therefore we pose the following question: Can the hybrid model succeed where the other two (FSUGold and NL3) have failed? Recall that the hybrid model was built with the explicit purpose of having a "low" incompressibility coefficient of $K_{0} \approx 230 \mathrm{MeV}$ and a "large" (and negative) asymmetric term of $K_{\tau}=-532 \mathrm{MeV}$ (see Table III), unlike FSUGold where both $K_{0}$ and $\left|K_{\tau}\right|$ are low, and unlike NL3

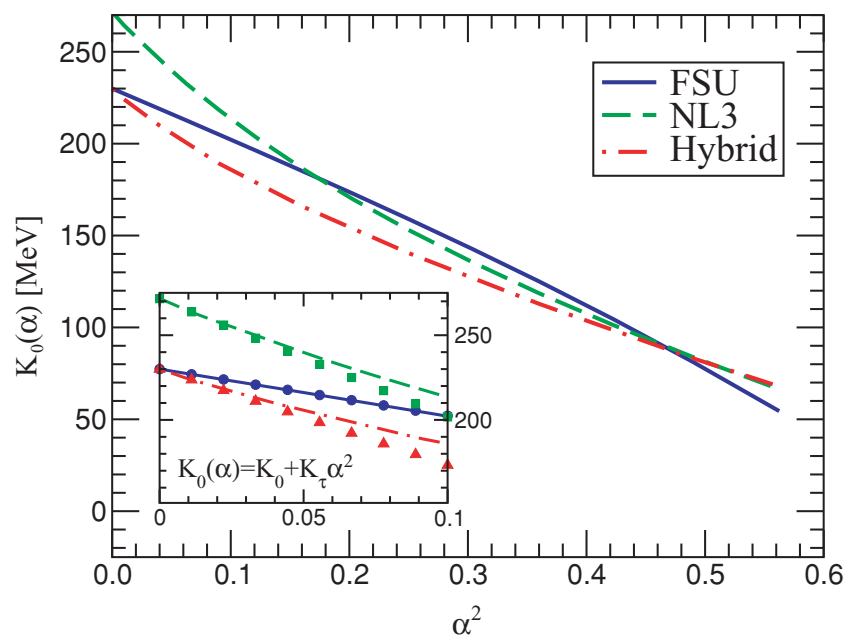

FIG. 6. (Color online) Same as Fig. 4, but for the incompressibility coefficient.

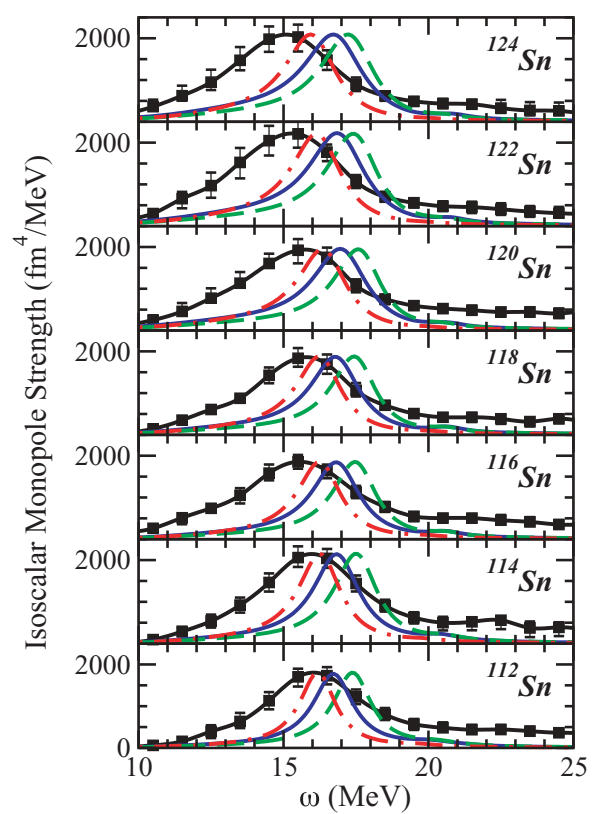

FIG. 7. (Color online) Comparison between the distribution of isoscalar monopole strength in all neutron-even ${ }^{112} \mathrm{Sn}-{ }^{124} \mathrm{Sn}$ isotopes extracted from experiment [6,7] (black solid squares) and the theoretical predictions of the FSUGold (blue solid line), NL3 (green dashed line), and hybrid (red dot-dashed line) models.

where both $K_{0}$ and $\left|K_{\tau}\right|$ are high. Thus, as in Ref. [8], the distribution of isoscalar monopole strength for the even- $A$ tin isotopes-from ${ }^{112} \mathrm{Sn}$ up to ${ }^{124} \mathrm{Sn}$ - was computed in a relativistic random-phase approximation (RPA). Details of the method may be found in Ref. [35].

Figures 7-9 are reminiscent of those published in Ref. [8], but in the present case results are also included for the hybrid model. It is clear from Fig. 7 that the experimental distribution

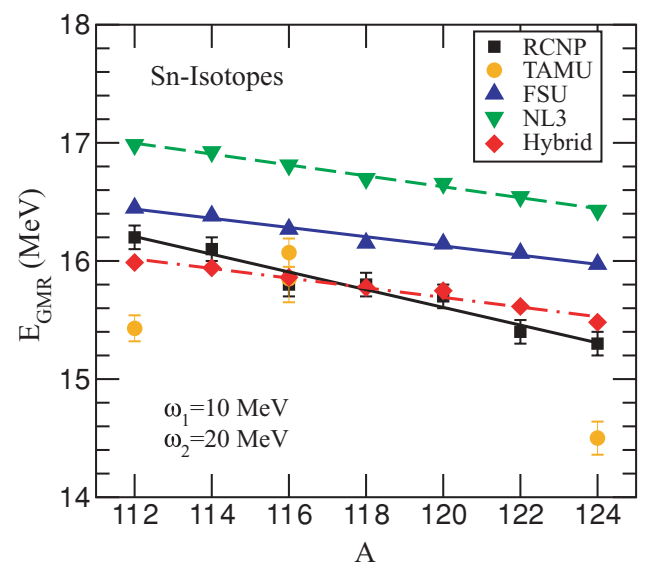

FIG. 8. (Color online) Comparison between the GMR centroid energies $\left(m_{1} / m_{0}\right)$ in all neutron-even ${ }^{112} \mathrm{Sn}-{ }^{124} \mathrm{Sn}$ isotopes extracted from experiment [6,7] (black solid squares) and the theoretical predictions of the FSUGold (blue up-triangles), NL3 (green downtriangles), and hybrid (red dot-dashed line) models. Also shown (filled gold circles) are experimental results from the TAMU group $[30,36,37]$ for the cases of ${ }^{112} \mathrm{Sn},{ }^{116} \mathrm{Sn}$, and ${ }^{124} \mathrm{Sn}$. 


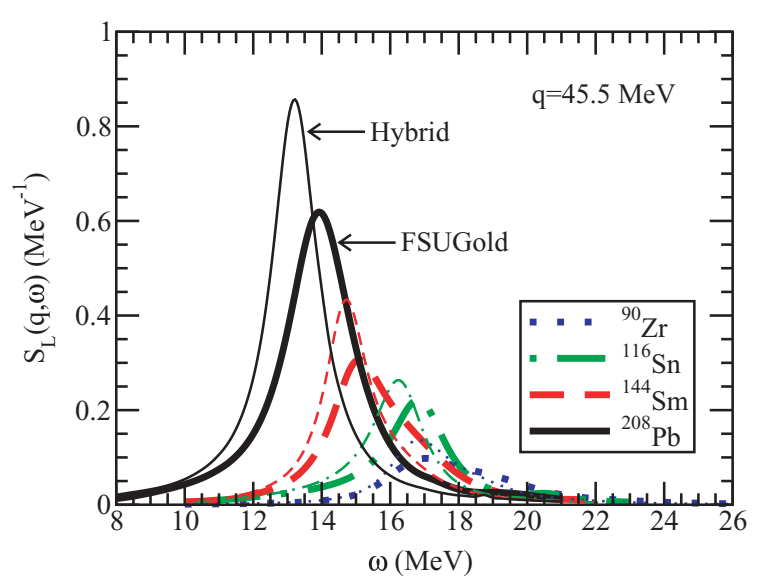

FIG. 9. (Color online) Distribution of isoscalar monopole strength for ${ }^{90} \mathrm{Zr}$, ${ }^{116} \mathrm{Sn},{ }^{144} \mathrm{Sm}$, and ${ }^{208} \mathrm{~Pb}$ as predicted for the FSUGold (thick lines) and hybrid (thin lines) models. Experimental centroid energies for these nuclei were reported in Ref. [30] and have been tabulated along with the theoretical predictions in Table IV.

of strength in the tin isotopes is best reproduced by the hybrid model. Note that RPA distributions of strength fail to account for the full-escape-plus-spreading — width of the resonance. Whereas the RPA calculation accounts properly for the escape width (i.e., the coupling to the continuum is treated exactly), it fails to account for its spreading component, as this one is related to configurations significantly more complex than those included in the RPA approach.

On the other hand, the RPA approach is sophisticated enough to reproduce the experimental centroid energy of the resonance. As it was done experimentally, the centroid energy was computed from the ratio of the $m_{1}$ to the $m_{0}$ moment. That is,

$$
E_{\mathrm{GMR}}=\frac{m_{1}}{m_{0}}=\frac{\int_{\omega_{1}}^{\omega_{2}} \omega S_{\mathrm{L}}(q, \omega) d \omega}{\int_{\omega_{1}}^{\omega_{2}} S_{\mathrm{L}}(q, \omega) d \omega},
$$

where $S_{\mathrm{L}}(q, \omega)$ is the distribution of strength. The integration limits have been fixed at $\omega_{1}=10 \mathrm{MeV}$ and $\omega_{2}=20 \mathrm{MeV}$, respectively, and the integrals have been evaluated at the small momentum transfer of $q \sim 0.23 \mathrm{fm}^{-1}$ (or $q \sim 45 \mathrm{MeV}$ ) [8]. The theoretical predictions for $E_{\mathrm{GMR}}$ in the tin isotopes are displayed in Fig. 8 in comparison with the experimental data from the Research Center for Nuclear Physics (RCNP), Osaka University [6,7], and Texas A\&M University (TAMU) [30,36, 37].
Although the FSUGold and hybrid models share the same value of the incompressibility coefficient in symmetric nuclear matter, the hybrid model provides a softer distribution of strength because of its largest (negative) asymmetric term $K_{\tau}$ (see Table III). Ultimately, this result hinges on the fact that the hybrid model has, as NL3, a significantly stiffer symmetry energy. All in all, the agreement between the hybrid model and experiment is adequate, although the model-indeed all models-could benefit from a steeper slope in the change of the centroid energy $E_{\mathrm{GMR}}$ with mass number $A$. To test the robustness of our results, we have used an improved version of the hybrid model that was obtained through a slight adjustment of the scalar mass $m_{s}(508.194 \rightarrow 494 \mathrm{MeV})$ and the corresponding coupling constant $g_{s}^{2}(106.2575 \rightarrow$ 100.4048, which yields the same $g_{s}^{2} / \mathrm{m}_{s}^{2}$ value and ensures that all of the properties of the EOS of infinite nuclear matter remain unaltered). This mild adjustment yields better ground-state masses for a few selected nuclei $\left({ }^{40} \mathrm{Ca},{ }^{90} \mathrm{Zr}\right.$, and $\left.{ }^{208} \mathrm{~Pb}\right)$, albeit at the expense of slightly worse charge radii. Yet the GMR energies for the $\mathrm{Sn}$ isotopes get softened by at most $1.5 \%$. This confirms one of the main results of this work, namely, that even a model with a soft $K_{0}$ (such as FSUGold) and a stiff $K_{\tau}$ (such as NL3) is unable to fully account for the rapid softening of the experimental GMR energies in the tin isotopes.

Therefore, where does theory stand with respect to experiment? Unquestionably, a hybrid model having a soft incompressibility coefficient but a stiff symmetry energy leads to a significant improvement when compared with the experiment on the tin isotopes [6,7]. Unfortunately, the hybrid model does not fare as well against other observables (see Table IV). First, the hybrid model predicts a GMR centroid energy in ${ }^{208} \mathrm{~Pb}$ of $E_{\mathrm{GMR}}=13.27 \mathrm{MeV}$ (or $13.16 \mathrm{MeV}$ if we use the hybrid model with $m_{s}=494 \mathrm{MeV}$ ), significantly lower than the experimental value of $14.17 \pm 0.28 \mathrm{MeV}$ [30]; in contrast, the FSUGold model gives a value of $E_{\mathrm{GMR}}=$ $14.04 \mathrm{MeV}$ [5] that is consistent with experiment. Note that similar trends have recently been reported by Avdeenkov and collaborators [10]. To appreciate the significant softening of the hybrid model relative to FSUGold, the distribution of isoscalar monopole strength for ${ }^{90} \mathrm{Zr}$, ${ }^{16} \mathrm{Sn},{ }^{144} \mathrm{Sm}$, and ${ }^{208} \mathrm{~Pb}$ is displayed in Fig. 9. Second, as we have argued earlier, a large negative asymmetry term $K_{\tau}$ requires a large positive value of the asymmetry pressure $L$. However, models with a stiff symmetry energy appear to be in conflict with model-independent predictions for the equation of state of pure neutron matter at low densities [26,27,38,39]. Finally, note that a stiff symmetry energy at densities below saturation also

TABLE IV. GMR centroid energies $\left(E_{\mathrm{GMR}} \equiv m_{1} / m_{0}\right.$ in $\left.\mathrm{MeV}\right)$ obtained from the distribution of monopole strength integrated over the $\omega_{1}-\omega_{2}$ range for those nuclei studied in Ref. [30]; $\alpha$ represents their respective neutron-proton asymmetry.

\begin{tabular}{lcccccr}
\hline \hline Nucleus & $\alpha$ & $\omega_{1}-\omega_{2}$ & Experiment & FSU & NL3 & Hybrid \\
\hline${ }^{90} \mathrm{Zr}$ & 0.111 & $10-26$ & $17.89 \pm 0.20$ & 17.98 & 18.62 & 17.47 \\
${ }^{116} \mathrm{Sn}$ & 0.138 & $10-23$ & $16.07 \pm 0.12$ & 16.58 & 17.10 & 16.02 \\
${ }^{144} \mathrm{Sm}$ & 0.139 & $10-22$ & $15.39 \pm 0.28$ & 15.64 & 16.14 & 15.07 \\
${ }^{208} \mathrm{~Pb}$ & 0.212 & $8-21$ & $14.17 \pm 0.28$ & 14.04 & 14.32 & 13.27 \\
\hline \hline
\end{tabular}


seems to be disfavored by heavy-ion data [19-22], although in this case the model dependence may be more significant, and the results are not always without some controversy [40,41].

\section{CONCLUSIONS}

The saturation properties of neutron-rich matter were studied as functions of the neutron-proton asymmetry within the framework of relativistic mean-field models. We observed that infinite nuclear matter continues to saturate up to values of the neutron-proton asymmetry of the order of $\alpha \equiv(N-Z) / A \lesssim$ 0.75 . Moreover, it was found quite generally that as infinite nuclear matter departs from the symmetric $(N=Z)$ limit, the saturation density lowers, the binding energy weakens, and the nuclear incompressibility softens.

The manuscript was organized around three main themes: (1) the use of accurately calibrated relativistic mean-field models to extract the saturation properties of neutron-rich matter directly from numerical computations and the comparison of these numerical results against approximate analytic approaches, (2) the use of the same models to compute the distribution of isoscalar-monopole strength in various nuclei, and (3) the comparison of these theoretical predictions against the experimentally measured GMR energies.

To make contact between the equation of state of bulk neutron-rich matter and GMR energies on finite nuclei, the incompressibility of infinite neutron-rich matter was parametrized in terms of two bulk parameters, namely, $K_{0}$ and $K_{\tau}$, with the former being the incompressibility coefficient of symmetric matter and the latter parametrizing the (small) deviations from the symmetric limit [see Eq. (18c)]. Note that never in the manuscript did we rely on semiempirical (liquid-drop-like) formulas to extract properties of infinite matter from extrapolating finite-nuclei results to the $A \rightarrow \infty$ limit. In this manner, we followed the time-honored tradition initiated by Blaizot and collaborators [1,2] of demanding that the values of both $K_{0}$ and $K_{\tau}$ be those extracted from a consistent theoretical model that successfully reproduces the experimental GMR energies of a variety of nuclei.

As part of the first theme, the evolution with neutronproton asymmetry of the saturation density, binding energy per nucleon, and incompressibility coefficient were extracted from a fit to the numerically generated equation of state. Once these properties were extracted, their dependence on the neutron-proton asymmetry $\alpha$ was captured through a simple parametrization in powers of $\alpha^{2}$ with no reliance on the parabolic approximation of Eq. (2) nor on an expansion involving bulk-model parameters, as in Eq. (11). Having completed this numerical procedure, we explored the possibility of reproducing the exact numerical results from analytic expansions based on a few bulk parameters of the equation of state determined at normal nuclear-matter saturation density [see Eqs. (18)]. For all three bulk properties-the saturation density, the binding energy per nucleon, and the incompressibility coefficient - the analytic values were in close agreement with those computed numerically. This seems to be a robust result, as it holds for all three (FSUGold, NL3, and hybrid) models; see Table III. Thus, we concluded that the analytic expres- sions are not only insightful but also quantitatively accurate. Particularly interesting is the case of the incompressibility coefficient $K_{\tau}$ that is given as the sum of three potentially "large and canceling" contributions. However, we found that one of these three terms- the slope of the symmetry energy $L$-dominates $K_{\tau}$ [see Eq. (18c)], thereby making sensitive cancellations unlikely. This result revealed an interesting correlation between $K_{\tau}$ and $L$ that may be further explored by the upcoming parity radius experiment (PREx) at the Thomas Jefferson National Accelerator Facility. PREx promises to measure the neutron radius of ${ }^{208} \mathrm{~Pb}$ accurately and model independently via parity-violating electron scattering $[42,43]$. PREx will provide a unique experimental constraint on the density dependence of the symmetry energy due its strong correlation to the neutron radius (or neutron skin thickness) of heavy nuclei [44].

To test the predictions of these three models, contact had to be made with available experimental data on finite nuclei. Thus, the distribution of isoscalar monopole strength was computed for a variety of nuclei in a consistent RPA approach [35]. In particular, the main motivation behind introducing the hybrid model was the inability of both FSUGold and NL3 to reproduce the recently measured GMR energies along the isotopic chain in tin [6,7]. By adopting a relatively small value for the incompressibility coefficient in symmetric matter $\left(K_{0}=230 \mathrm{MeV}\right)$ together with a fairly large negative value for its leading deviation from the symmetric limit $\left(K_{\tau} \approx\right.$ $-530 \mathrm{MeV}$ ), we constructed a hybrid model with a significantly softer incompressibility coefficient for neutron-rich matter. Such a softening indeed produced a significant improvement vis-à-vis the experimental data on the Sn isotopes; see Fig. 8. Whereas FSUGold and NL3 overestimate the centroid energy in ${ }^{124} \mathrm{Sn}$ by about 0.7 and $1.0 \mathrm{MeV}$, respectively, the hybrid model falls within $0.1 \mathrm{MeV}$ of the experimental data. Indeed, the predictions of the hybrid model fall within $0.1 \mathrm{MeV}$ of the experimental data for the full isotopic chain if one takes into account the uncertainties in the data. However, although the improvement in the case of the $\mathrm{Sn}$ isotopes is significant and unquestionable, an important problem remains: the hybrid model underestimates the GMR centroid energy in ${ }^{208} \mathrm{~Pb}$ - the heaviest doubly magic nucleus — by almost $1 \mathrm{MeV}$. This suggests that the rapid softening with neutron excess predicted by the hybrid model may be unrealistic.

Thus, where does theory stand with respect to experiment? One possibility, given that FSUGold reproduces the centroid energy in both ${ }^{90} \mathrm{Zr}$ (with $\alpha=0.11$ ) and ${ }^{208} \mathrm{~Pb}$ (with $\alpha=0.21$ ), is that its predictions for $K_{0}$ and $K_{\tau}$ are reliable, but that its failure to reproduce the GMR energies in tin is due to missing physics unrelated to the incompressibility of neutronrich matter. We feel inclined to favor this possibility for two main reasons. First, the missing physics may be to some extent related to the open-shell structure of the tin isotopes, a property that makes pairing correlations essential and endows Tin with its superfluid character. Support in favor of this scenario has been recently presented in Ref. [45], where a surface pairing force was used to bring theory much closer to experiment, at least from ${ }^{112} \mathrm{Sn}$ to ${ }^{120} \mathrm{Sn}$. Second, the large and negative value suggested from the experimental extraction of $K_{\tau}$ may be at odds with theoretical constraints deduced from the behavior of 
dilute Fermi gases that seem to suggest a moderate value for the pressure of pure neutron matter at saturation density [27]. (Note that the pressure of pure neutron matter, or equivalently the slope of the symmetry energy $L$, largely determines the behavior of $K_{\tau}$.) This suggests that the value of $K_{\tau}=-550 \pm$ $100 \mathrm{MeV}$ inferred from experiment [6,7] may suffer from the same ambiguities already encountered in earlier attempts to extract the incompressibility coefficient of infinite matter from finite-nuclei extrapolations. Yet the final resolution of the question "why is tin so soft?" awaits further theoretical insights.

\section{ACKNOWLEDGMENTS}

The authors are grateful to Professors M. Di Toro and H. Sagawa for useful discussions. This work was supported in part by grants from the US Department of Energy DE-FD0592ER40750, FIS2008-01661 from MEC (Spain) and FEDER, and 2005SGR-00343 from Generalitat de Catalunya, and by the Spanish Consolider-Ingenio 2010 Programme CPAN CSD2007-00042.

\section{APPENDIX: NOMENCLATURE AND TERMINOLOGY}

In this section, we address what we perceive as a confusing state of affairs with regard to the nomenclature used to characterize the symmetry energy. First, we note that no uniform terminology exists even to denote the neutron-proton asymmetry coefficient. Indeed, the symbols $I$ [46], $\alpha$ [22,47, $48], \beta[32]$, and $\delta[9,19,49]$ are all used in the literature to denote the neutron-proton asymmetry coefficient $(N-Z) / A$ of asymmetric nuclear matter. Second, and perhaps even more confusing, is the myriad of different symbols used to refer to the same bulk properties. For example, all of the following expressions may be found in the literature $[46,47]$ :

$$
\begin{aligned}
\mathcal{S}(\rho) & =J+L x+\frac{1}{2} K_{\mathrm{sym}} x^{2}+\cdots \\
& =a_{4}+\frac{L}{3}\left(\frac{\rho-\rho_{0}}{\rho_{0}}\right)+\frac{K_{\mathrm{sym}}}{18}\left(\frac{\rho-\rho_{0}}{\rho_{0}}\right)^{2}+\cdots \\
& =a_{4}+\frac{P_{0}}{\rho_{0}^{2}}\left(\rho-\rho_{0}\right)+\frac{\Delta K_{0}}{18 \rho_{0}^{2}}\left(\rho-\rho_{0}\right)^{2}+\cdots,
\end{aligned}
$$

where $x=\left(\rho-\rho_{0}\right) / 3 \rho_{0}$. Moreover, another plausible expansion of the symmetry energy may be around the equilibrium Fermi momentum [49]. That is,

$$
\mathcal{S}(\rho)=\tilde{J}+\tilde{L} y+\frac{1}{2} \widetilde{K}_{\mathrm{sym}} y^{2}+\cdots,
$$

where the deviation from the equilibrium Fermi momentum has been parametrized in terms of the dimensionless parameter $y$ defined as

$$
y \equiv \frac{k_{\mathrm{F}}-k_{\mathrm{F}}^{0}}{k_{\mathrm{F}}^{0}} .
$$

Recall that the Fermi momentum and the baryon density are related by the following expression:

$$
\rho=\frac{2 k_{\mathrm{F}}^{3}}{3 \pi^{2}} .
$$

One potential confusion between the two different expansions of the symmetry energy (in terms of either $x$ or $y$ ) is that in the presence of a linear term (such as $L$ ) the coefficients are in general not equal. Indeed, the various bulk coefficients are related as follows:

$$
\begin{aligned}
\tilde{J} & =J, \\
\tilde{L} & =L, \\
\widetilde{K}_{\mathrm{sym}} & =K_{\text {sym }}+2 L .
\end{aligned}
$$

That is, at order $y^{2}$ and higher, the expansion coefficients in terms of the Fermi momentum $y$ differ from the corresponding ones used in an expansion in terms of the density $x$.

It is also common practice to express the finite nucleus incompressibility coefficient $\left(K_{A}\right)$ by means of a liquid-droplike mass formula [2,6,9,48-51], which highlights the physical meaning of the various contributions to $K_{A}$. That is,

$$
\begin{aligned}
K_{A}= & K_{\mathrm{vol}}+K_{\mathrm{surf}} A^{-1 / 3}+K_{\tau}\left(\frac{N-Z}{A}\right)^{2} \\
& +K_{\operatorname{Coul}} \frac{Z^{2}}{A^{4 / 3}}+\cdots .
\end{aligned}
$$

In some works, the coefficient $K_{\tau}$ is denoted by $K_{\text {asy }}[19,22,48]$ or $K_{\mathrm{vs}}[50,51]$. To add to the confusion in notation, in the original contributions by Blaizot and collaborators $[1,2,49]$ the term $K_{\text {sym }}$ was used instead of $K_{\tau}$ in Eq. (A6). It appears that at present, $K_{\text {sym }}$ has been "universally" adopted to refer to the curvature of the symmetry energy at saturation density, as in Eq. (7).

In summary, we adopt the following convention in the present manuscript - and hopefully in all future works. The energy per particle of asymmetric nuclear matter is denoted as

$$
\begin{aligned}
\mathcal{E}(\rho, \alpha)= & \mathcal{E}_{\mathrm{SNM}}(\rho)+\mathcal{S}(\rho) \alpha^{2}+\mathcal{O}\left(\alpha^{4}\right) \\
= & \left(\varepsilon_{0}+\frac{1}{2} K_{0} x^{2}+\frac{1}{6} Q_{0} x^{3}+\cdots\right) \\
& +\left(J+L x+\frac{1}{2} K_{\mathrm{sym}} x^{2}\right. \\
& \left.+\frac{1}{6} Q_{\mathrm{sym}} x^{3}+\cdots\right) \alpha^{2}+\mathcal{O}\left(\alpha^{4}\right),
\end{aligned}
$$

where the dimensionless parameters $x$ and $\alpha$ characterize the deviations from saturation density and from the symmetric limit, respectively. That is,

$$
\begin{gathered}
x \equiv \frac{\rho-\rho_{0}}{3 \rho_{0}}, \\
\alpha \equiv \frac{N-Z}{A} .
\end{gathered}
$$

Finally, the quantities $\rho_{\tau}, \varepsilon_{\tau}$, and $K_{\tau}$, have been introduced to denote, respectively, the evolution with the neutron-proton asymmetry of the saturation density, the energy per particle, and the incompressibility coefficient of infinite neutron-rich matter. That is,

$$
\begin{gathered}
\rho_{0}(\alpha)=\rho_{0}+\rho_{\tau} \alpha^{2}+\mathcal{O}\left(\alpha^{4}\right), \\
\varepsilon_{0}(\alpha)=\varepsilon_{0}+\varepsilon_{\tau} \alpha^{2}+\mathcal{O}\left(\alpha^{4}\right), \\
K_{0}(\alpha)=K_{0}+K_{\tau} \alpha^{2}+\mathcal{O}\left(\alpha^{4}\right) .
\end{gathered}
$$


[1] J. P. Blaizot, D. Gogny, and B. Grammaticos, Nucl. Phys. A265, 315 (1976).

[2] J. P. Blaizot, J. F. Berger, J. Dechargé, and M. Girod, Nucl. Phys. A591, 435 (1995).

[3] J. M. Pearson, Phys. Lett. B271, 12 (1991).

[4] S. Shlomo and D. H. Youngblood, Phys. Rev. C 47, 529 (1993).

[5] B. G. Todd-Rutel and J. Piekarewicz, Phys. Rev. Lett. 95, 122501 (2005).

[6] T. Li et al., Phys. Rev. Lett. 99, 162503 (2007).

[7] U. Garg et al., Nucl. Phys. A788, 36 (2007).

[8] J. Piekarewicz, Phys. Rev. C 76, 031301(R) (2007).

[9] H. Sagawa, S. Yoshida, G.-M. Zeng, J.-Z. Gu, and X.-Z. Zhang, Phys. Rev. C 76, 034327 (2007).

[10] A. Avdeenkov et al., Phys. Rev. C 79, 034309 (2009).

[11] B. D. Serot and J. D. Walecka, Adv. Nucl. Phys. 16, 1 (1986).

[12] B. D. Serot and J. D. Walecka, Int. J. Mod. Phys. E 6, 515 (1997).

[13] C. J. Horowitz and J. Piekarewicz, Phys. Rev. Lett. 86, 5647 (2001).

[14] B. G. Todd and J. Piekarewicz, Phys. Rev. C 67, 044317 (2003).

[15] A. Bohr and B. R. Mottelson, Nuclear Structure (World Scientific, Singapore, 1998), Vol. I.

[16] M. B. Tsang, W. A. Friedman, C. K. Gelbke, W. G. Lynch, G. Verde, and H. Xu, Phys. Rev. Lett. 86, 5023 (2001).

[17] M. B. Tsang et al., Phys. Rev. Lett. 92, 062701 (2004).

[18] D. V. Shetty et al., Phys. Rev. C 70, 011601(R) (2004).

[19] B.-A. Li and L.-W. Chen, Phys. Rev. C 72, 064611 (2005).

[20] D. V. Shetty, S. J. Yennello, and G. A. Souliotis, Phys. Rev. C 75, 034602 (2007).

[21] D. V. Shetty, S. J. Yennello, and G. A. Souliotis, Phys. Rev. C 76, 024606 (2007).

[22] B.-A. Li, L.-W. Chen, and C. M. Ko, Phys. Rep. 464, 113 (2008).

[23] A. Klimkiewicz et al., Phys. Rev. C 76, 051603(R) (2007).

[24] L. Trippa, G. Colò, and E. Vigezzi, Phys. Rev. C 77, 061304(R) (2008).

[25] M. Centelles, X. Roca-Maza, X. Vinas, and M. Warda, Phys. Rev. Lett. 102, 122502 (2009).

[26] A. Schwenk and C. J. Pethick, Phys. Rev. Lett. 95, 160401 (2005).
[27] J. Piekarewicz, Phys. Rev. C 76, 064310 (2007).

[28] G. A. Lalazissis, J. Konig, and P. Ring, Phys. Rev. C 55, 540 (1997).

[29] G. A. Lalazissis, S. Raman, and P. Ring, At. Data Nucl. Data Tables 71, 1 (1999).

[30] D. H. Youngblood, H. L. Clark, and Y.-W. Lui, Phys. Rev. Lett. 82, 691 (1999).

[31] H. Mueller and B. D. Serot, Nucl. Phys. A606, 508 (1996).

[32] W. Zuo, I. Bombaci, and U. Lombardo, Phys. Rev. C 60, 024605 (1999).

[33] H. Heiselberg and M. Hjorth-Jensen, Phys. Rep. 328, 237 (2000).

[34] S. K. Samaddar, J. N. De, X. Vinas, and M. Centelles, Phys. Rev. C 78, 034607 (2008).

[35] J. Piekarewicz, Phys. Rev. C 64, 024307 (2001).

[36] D. H. Youngblood, Y. W. Lui, H. L. Clark, B. John, Y. Tokimoto, and X. Chen, Phys. Rev. C 69, 034315 (2004).

[37] Y. W. Lui, D. H. Youngblood, Y. Tokimoto, H. L. Clark, and B. John, Phys. Rev. C 70, 014307 (2004).

[38] C. J. Horowitz and A. Schwenk, Nucl. Phys. A776, 55 (2006).

[39] S. Kowalski et al., Phys. Rev. C 75, 014601 (2007).

[40] D. V. Shetty et al., arXiv:nucl-ex/0603016.

[41] A. Ono, P. Danielewicz, W. A. Friedman, W. G. Lynch, and M. B. Tsang, arXiv:nucl-ex/0507018.

[42] C. J. Horowitz, S. J. Pollock, P. A. Souder, and R. Michaels, Phys. Rev. C 63, 025501 (2001).

[43] R. Michaels, P. A. Souder, and G. M. Urciuoli (2005), http://hallaweb.jlab.org/parity/prex.

[44] B. A. Brown, Phys. Rev. Lett. 85, 5296 (2000).

[45] J. Li, G. Colò, and J. Meng, Phys. Rev. C 78, 064304 (2008).

[46] V. Baran, M. Colonna, V. Greco, and M. Di Toro, Phys. Rep. 410, 335 (2005).

[47] R. J. Furnstahl, Nucl. Phys. A706, 85 (2002).

[48] L.-W. Chen, C. M. Ko, and B.-A. Li, Phys. Rev. C 76, 054316 (2007).

[49] J. P. Blaizot, Phys. Rep. 64, 171 (1980).

[50] T. v. Chossy and W. Stocker, Phys. Rev. C 56, 2518 (1997).

[51] S. K. Patra, M. Centelles, X. Vinas, and M. Del Estal, Phys. Rev. C 65, 044304 (2002). 